\title{
The anthropic view of nucleosynthesis
}

Our understanding of the Universe may be shaped in part by the circumstance that our knowledge of it derives exclusively from people like ourselves, but what if very different forms of life exist?

THE anthropic principle may be generally known, but that does not mean that it is generally accepted - or even generally understood. And that may be forgivable, for there is a sense in which the anthropic principle does no more than make a virtue of tautology.

In its weakest form, the principle amounts simply to the remark that the only known sources of the observations on which rests our present understanding of the Universe are people like ourselves, members of a species emergent only relatively recently from a long and complicated process of evolution. What this implies is that, of all possible universes, people like ourselves can observe only those old enough to allow for, say, 3,000 million years of natural selection and which also provide the other conditions required for evolution, a reasonable constant ambient temperature for example. So is there much more to say than that the Universe we see is the only one we could see?

The more constructive way to put the argument is to say that because observations of the Universe are collected (so far as is known) only by people, and because the evolution of people requires certain conditions to be met by the physical world, the mere existence of living things makes it possible to say something significant about the Universe as a whole. As will emerge, there is a logical flaw in that element of the anthropic principle, but at least it has the virtue of suggesting that a simple listing of the conditions required for life may have a bearing not only on the question how life began, but on that of how the Universe is constructed as well.

The obvious starting point is the time occupied by biological evolution, which is at least the time elapsed since the earliest forms of microbial life-forms recognizable in Proterozoic rock formations, say 2,700 million years or more. (Those who believe in panspermia - the population of the surface of the Earth by life-forms from space - allow themselves more room for manoeuvre in dealing with the problem of time, but at the cost of straining the attention of their listeners.) As more is learned of the early stages of evolution, and of the physical conditions on the surface of the Earth in early times, it will no doubt be possible to define evermore stringently the conditions that must be satisfied for the emergence of lifeforms of any kind, let alone of people, and thus to say even more about the properties of the whole Universe.

Without mentioning the anthropic principle as such, R. E. Davies and R. H. Koch from the University of Pennsylvania have carried the argument one big step backwards in time by asking what conditions must have been satisfied so that the Solar System should contain the chemical elements required to sustain life (Phil. Trans. R. Soc. 334B, 391-403; 1991). Their starting point is the observation that all lifeforms consist not only of carbon, oxygen and hydrogen, but of elements such as phosphorus (as in DNA), iron (as in haemoglobin) and even cobalt (as in vitamin $B_{12}$ ). So what would have been required of nucleosynthesis, before the formation of the Sun, so that life could afterwards have emerged as it has done (on one planet of the Solar System?)

The unavoidable starting-point is the assumption that the primordial material consists largely of hydrogen mixed with a tenth as many atoms of helium. All other elements incorporated into living things must have been made at a later stage by nucleosynthesis in supernovae and other kinds of stars. While supernovae are the best known sources of material such as this, it is crucial that some elements fluorine, for example - are made predominantly on the surfaces of white dwarf stars bound into binary systems in which a larger companion has lost material to the white dwarf.

That conclusion has an immediate bearing on the origin of the material from which the Solar System is made: some of it must have come from earlier supernova explosions and some from the burning of nitrogen nuclei on the surfaces of dwarf stars in binary systems. In other words, the fluorine problem by itself is a proof that the material of which the Solar System is formed came from various stellar sources. That is not surprising. Supernova explosions may produce debris consisting of one or more solar masses of material, but that is necessarily scattered in all directions, so that the formation of a new solar system would require contributions from at least several exploded stars.

The chief value of what Davies and Koch have done may well rest on their compilation of data on the elements involved in living things in a way that must stimulate others to constructive speculation. There is, for example, the observation that even humble E.coli depends on seventeen elements (counting hydrogen, oxygen and carbon), compared with 26 for people. There is also the curious business of why it should be that some eukaryotes concentrate elements such as mercury and cadmium relative to their environment and have then had to evolve detoxifying systems so as to avoid the ill-effects. But with all that said, Davies and Koch are evidently most of all wrapped up with the cosmic significance of their data.

This is how the argument goes. As things are, something like 1.9 per cent of the material of which the Galaxy was formed 20,000 million years ago (or half as long ago, depending on the true value of Hubble's Constant) has been converted by supernova explosions and other means into atoms heavier than helium. Using rough estimates for the efficiency with which supernova explosions effect nucleosynthesis, they conclude that there must have been an average of one supernova explosion every three years since the beginning of the Galaxy and, because the present rate is much smaller, that most of the nucleosynthesis must have happened early in its history. In short, Davies and Koch say, every part of the Galaxy contributed to the formation of the Solar System 5,000 million years ago.

Although the anthropic principle is not mentioned, the bearing of this argument about the origins of the Solar System should be clear: it is not simply that enough time must have passed to allow for the evolution of people, but that that will not happen unless there has been enough nucleosynthesis within some self-contained structure such as a galaxy to make solar systems capable of sustaining life. In short, the only kinds of universes that will be seen by the likes of us must be universes in which there are galaxies whose separate stars are continually refashioning themselves through supernovae.

So what is the logical flaw? Decades ago, a British astronomer of conventional mien was explaining why there could be no other inhabited planet than the Earth to an audience that included the then-youthful Thomas Gold, now to be found at Cornell University. When the speaker had assumed for the umpteenth time that all life is terrestrial, Gold delivered the stage whisper, "Bloody fool, they might look like rocks!"

John Maddox 